\title{
Genetic Algorithm Applied to Discrete Optimization of Band-Pass Microwave Filter
}

\author{
Darko Ninković and Dragan Olćan, Member, IEEE
}

\begin{abstract}
We propose a design approach for band-pass microwave filters based on optimization of discrete-valued parameters of filters using an implementation of $(1+(\lambda, \lambda))$ genetic algorithm. If the filter is made of SMD components then only discrete values are allowed. The proposed design approach yields a solution with feasible components values in the end, without further need for tuning and adjustments of component values that is needed in the case when real numbers are used during optimization. The optimization setup is run for 20 times and obtained (suboptimal) solutions are presented. One of the best optimized solutions of a band-pass filter is chosen for further analysis. The found filter design is verified using a circuit solver including the yield analysis, 3D electromagnetic simulations and by measurements of the manufactured prototype. The presented results as well as the optimization run-time show that the proposed approach based on discrete optimization can be used to design microwave filters.
\end{abstract}

Keywords - Band-pass filter, discrete optimization, genetic algorithm.

\section{INTRODUCTION}

$\mathrm{M}$ ICROWAVE filters are incorporated in most RF and microwave devices and their design is a specialized field of microwave theory and techniques [1]-[6].

A standard procedure of microwave filter design with lumped-elements involves the optimization of component values. A typical design flow starts with optimization of elements that can have any value (real number) within a predefined range, i.e., the process yields real-valued components that are not necessarily commercially available. If the filter is to be made of discrete SMD components, then rounding to the nearest discrete value is needed as well as further fine-tuning of the discrete values [1]-[6].

The work presented here is an extension of [7].

We present a design approach for microwave filters based on discrete optimizations, i.e., the optimization works

Paper received April 13, 2021; accepted May 31, 2021. Date of publication July 31, 2021. The associate editor coordinating the review of this manuscript and approving it for publication was Prof. Branko Kolundžija.

This paper is revised and expanded version of the paper presented at the 28th Telecommunications Forum TELFOR 2020 [7].

The work presented here is supported in part by the Ministry of Education, Science and Technological Development of Republic of Serbia.

Darko M. Ninković (contact author) is with the School of Electrical Engineering, University of Belgrade, Bul. kralja Aleksandra 73, 11120 Belgrade, Serbia (phone: 381-11-3218386; e-mail: darko@etf.rs).

Dragan I. Olćan, is with the School of Electrical Engineering, University of Belgrade, Bul. kralja Aleksandra 73, 11120 Belgrade, Serbia (phone: 381-11-3218329; e-mail: olcan@etf.rs). exclusively with discrete values of (SMD) components available on the market. Since the final components of the microwave filter that we are designing can have only discrete values, the optimization (design) space is discrete, and there is a finite (but usually extremely large) number of possible solutions. Complete search of the optimization space might be possible for simpler designs, depending on the available computing resources. However, for real-life designs the complete search is infeasible due to the presentday computing resources. Nowadays, there is only a handful of options for the local discrete optimization of microwave filters both in the open literature and in commercial microwave software [8]-[11].

The lack of discrete optimizations for microwave filters motivated us to use a genetic algorithm (GA). It is a global optimization algorithm that can (theoretically) explore all parts of the optimization space. Moreover, its modifications for discrete-valued and continuous-valued optimizations are known and are being researched. This has motivated us to propose and use a discrete version of genetic algorithm for microwave filter design with a finite set of allowed component values.

Generally, the discrete-valued optimization of a microwave filter can be understood as an instance of combinatorial optimization or Boolean satisfiability problems, if all the optimization variables are encoded as vectors of bits.

In order to illustrate the proposed optimization approach and the encoding of microwave filter elements, we consider a band-pass filter that consists of six LC resonators (three connected in series and three connected in parallel), shown in Fig. 1. It is designed using the proposed discrete version of the GA that has similar operators of GA from [12]. However, the proposed algorithm belongs to the class of stochastic optimization algorithms, i.e., each optimization run yields a different solution (except in the case when the global optimum is found in every run). Finding different (suboptimal) solutions typically happens if the algorithm is stopped after a number of iterations or when the maximum optimization time is reached, rather than leaving GA to wait for the mutation that leads to convergence to the global optimum. Hence, in order to quantify the performances of the algorithm, we restarted the optimization algorithm multiple times and present results for cumulative minima and the average best solution found after a given number of filter analyses.

The optimization results are presented for the best found filter, as well as for the suboptimal solutions that can be of interest in practical applications. Namely, in engineering practice additional criteria can be introduced after the 
optimization to discern among suboptimal solutions (e.g., to minimize manufacturing time, to minimize the production costs, etc.)

The results of the optimization are cross-checked by further analyses of the solution using a circuit-solver [13] and full-wave electromagnetic simulations [14] and by measuring the fabricated prototype in the Laboratory for the microwave theory and techniques at the School of Electrical Engineering, University of Belgrade, Serbia.

The paper is organized as follows. In Section II we define the optimization problem - design of a band-pass microwave filter. Used genetic algorithm is outlined in Section III. In Section IV the (best) found solutions are analysed. The verification of the filter design with circuitsolver simulation is outlined in Section V. The full-wave (3D) electromagnetic simulation of the filter is presented in Section VI. The comparison between measurements, simulations and yield analysis of the fabricated prototype of the microwave filter is presented in Section VII. Finally, Section VIII concludes the paper.

\section{OptiMIZATION}

We consider a lumped-element band-pass filter that consists of three series LC resonators and three parallel LC resonators, shown in Fig. 1. Therefore, the topology of the filter is predefined. Nonetheless, the proposed methodology can be applied to any filter made of discrete components.

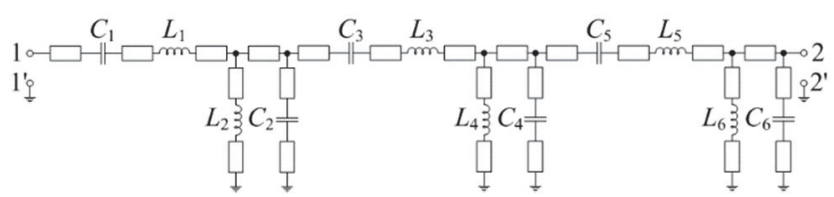

Fig. 1. Band-pass filter consisting of six LC resonators.

The objective is to optimize values of capacitors $C_{1}-C_{6}$ and inductors $L_{1}-L_{6}$ in order to achieve the predefined frequency response. For the considered filter the center frequency is $900 \mathrm{MHz}$ and it should have a $3 \mathrm{~dB}$ bandwidth of $100 \mathrm{MHz}$. The optimization function (cost function, or inverse fitness) to be minimized is defined as:

$$
f_{\text {opt }}\left(L_{1}, L_{2}, \ldots L_{6}, C_{1}, C_{2}, \ldots C_{6}\right)=\sum_{i=1}^{73} f\left(\text { freq }_{i}\right),
$$

where freq $_{i}$ are frequency points calculated as:

$$
\text { freq }_{i}=\left\{\begin{array}{l}
(300+10(i-1)) \mathrm{MHz}, 1 \leq i \leq 31 \\
(850+10(i-32)) \mathrm{MHz}, 32 \leq i \leq 42 \\
(1200+10(i-43)) \mathrm{MHz}, 43 \leq i \leq 73
\end{array},\right.
$$

and function $f\left(\right.$ freq $\left._{i}\right)$ is defined as:

$$
f\left(\text { freq }_{i}\right)=\left\{\begin{array}{l}
\left.\max \left(s_{21}\left(\text { freq }_{i}\right)[\mathrm{dB}]+20,0\right)\right), 1 \leq i \leq 31 \\
\left.\max \left(s_{11}\left(\text { freq }_{i}\right)[\mathrm{dB}]+20,0\right)\right), 32 \leq i \leq 42 . \\
\left.\max \left(s_{21}\left(\text { freq }_{i}\right)[\mathrm{dB}]+20,0\right)\right), 43 \leq i \leq 73
\end{array}\right.
$$

In order to perform fast analysis of the filter, the circuit analysis is done using a custom-made code based on the schematic shown in Fig. 1. The starting model of the filter consists of ideal capacitors, ideal inductors, lossless microstrip transmission lines and ideal connections between transmission lines. Thus, the model is described with nonlinear analytical expressions.

The substrate thickness is $0.5 \mathrm{~mm}$, and its relative permittivity is 4.6. The function for calculating $s$-parameters of the described model is coded in $\mathrm{C} / \mathrm{C}++$ for fast computations. It is based on equations for the impedances of the network and the impedance transformation along a lossless transmission line [2], [4].

The optimization (i.e., search or design) space for the inductors consists of 16 common SMD inductors values:

$$
\begin{aligned}
L_{[\mathrm{nH}]} \in\{1.2,1.5,2.2,5.1,5.8,8.2,10,15, & 22,27,30,39,47,82,100,150\}
\end{aligned},
$$

while the capacitors assume a discrete set of 32 common values:

$$
\begin{aligned}
& C_{[\mathrm{pF}]} \in\{0.5,1,1.2,1.5,1.8,2,2.2,2.7, \\
& 3.6,3.9,4.7,5.1,5.6,6,6.8,7, \\
& 8,8.2,9,10,12,15,16,18, \\
&20,22,24,27,30,33,36,47\}
\end{aligned}
$$

These sets are chosen by having in mind the commercially and typically available components.

Each of those 16 inductors values is encoded using a unique integer in the range $0-15$ and therefore every inductor variable is represented by 4 bits. Furthermore, 5 bits are used for encoding a single capacitor value. In order to encode a solution of this optimization problem as a single binary string, 54 bits are used. The first group of 24 bits in the string corresponds to six inductors values, and the next group of 30 bits stands for six capacitors values.

Consider an example of a binary string that represents a possible solution of the optimization problem shown in Fig.2: $00011101 \ldots 01011$. Starting 4 bits 0001 are a binary

\begin{tabular}{|c|c|c|c|c|c|c|c|c|c|c|c|}
\hline Binary string & 0 & 0 & 1 & 1 & 1 & 1 & $\ldots$ & 0 & 1 & 0 & 1 \\
\hline Component & & $\bar{L} L_{1}$ & & & $L_{2}$ & & ... & & & $\overline{c_{6}}$ & \\
\hline
\end{tabular}
representation of number 1 and as the result variable $L_{1}$ takes the $2^{\text {nd }}$ value from the set of possible inductors values (i.e., $1.5 \mathrm{nH}$ ). Next 4 bits encode the value of $L_{2}$, which takes the $14^{\text {th }}$ value from (4) that is $82 \mathrm{nH}$. The last 5 bits 01011, which is a binary representation of the decade number 11 , correspond to the value of $C_{6}$, which in the case of 01011 takes the $12^{\text {th }}$ of possible 32 values, which is in this case $5.1 \mathrm{pF}$.

Fig. 2. An example of a binary encoding of a solution.

The search space of the described optimization problem consists of $2^{54}$ (approximately $1.8 \cdot 10^{16}$ ) possible solutions. With such encoding, optimization function (1) is now defined on a discrete domain. With the presented encoding the problem of microwave filter design is transformed into the optimization problem that deals with binary strings as inputs, similar to Boolean satisfiability problems.

\section{DISCRETE IMPLEMENTATION OF GENETIC ALGORITHM}

In order to solve this optimization problem we use GA. It is a metaheuristic algorithm inspired by the laws of nature (selection and the survival of the fittest) [15], [16]. 
In the general case, GA consists of three operators: (1) selection, (2) crossover and (3) mutation. These operators are applied to the current generation of solutions. The final result of these operators is the new generation of solutions. The process repeats for multiple generations.

We implement a discrete version of $(1+(\lambda, \lambda))$ GA. Each step of the algorithm starts with a parent population of size one. The candidate solutions for the crossover are created by mutations. Namely, $\lambda$ candidates are created from the parent by mutation operator applied on each bit with the probability $p$, i.e., each bit in the encoding vector of the parent solution is flipped with probability $p$.

Then, the optimization functions are calculated for all candidate solutions for crossover. Those candidate solutions are compared with respect to the optimization function and the best candidate is selected for the crossover.

The algorithm uses crossover of probability $c$ to generate new generation of $\lambda$ offspring by combining the parameters (genetic information) of the starting parent (referred to as the parent 1 of the crossover) and the winning candidate of the mutation phase (referred to as the parent 2 of the crossover). Each bit of the offspring is taking the value of the corresponding bit of the parent 1 with probability $c$, and otherwise taking the value of the corresponding bit of the parent 2. An example of a crossover is shown in Fig. 3.

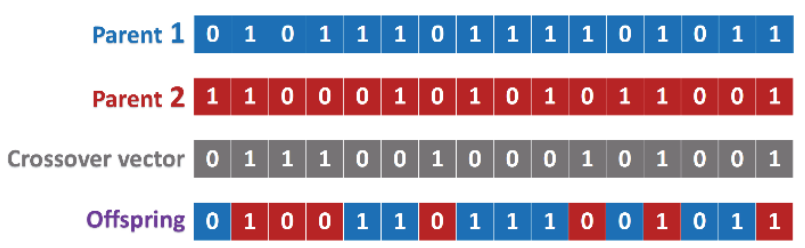

Fig. 3. An example of a crossover.

Finally, the fitness functions of the winning offspring of the crossover phase and the starting parent are compared and the better individual (i.e., the one with the lowest optimization function) is selected as a starting parent in the new step. One step of the algorithm consists of all described operations. Flow chart of the algorithm is shown in Fig. 4.

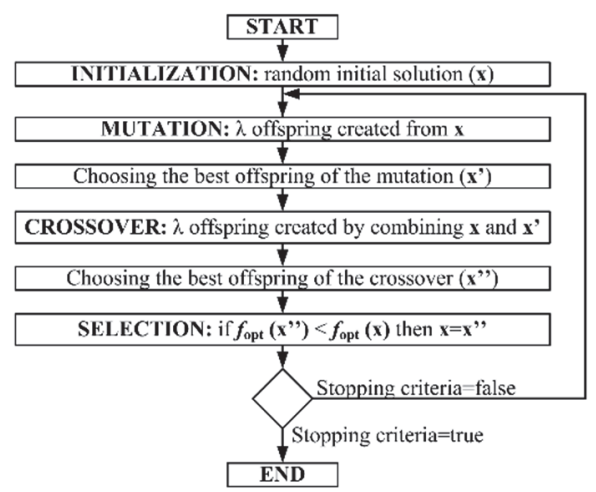

Fig. 4. Flow chart of the used GA.

Note that this implementation, due to the fact that it operates with a generation that has a single solution and generates new solutions using a random process, can be viewed also as an optimization algorithm between simulated annealing and GA. Nonetheless, the implementation is an instance of evolutionary strategy $(1+\lambda)-$ EA $[11]$.

In order to test the performances of the proposed algorithm we run the optimization for 25 times. For the algorithm parameters, we chose constant values of $p=0.1$, $c=0.5$ and $\lambda=100$. Those values lead to combining high mutation probability with crossover of the parent [12]. A large mutation rate should increase the probability of exploring other parts of optimization space, while the crossover should ensure that the parameters of the parent are not lost. The optimization process is terminated after 10000 steps since the algorithm typically stalls after that number of steps. If the algorithm stalls, the probability of generating a better solution is very small and from the point of rational use of computer resources, it is not feasible to continue the optimization. The progress of the optimization for each run (cumulative minimum), as well as the averaged progress (average cumulative minimum), are presented in Fig. 5, where the cost-function of the best solutions versus the number of algorithm steps are shown.

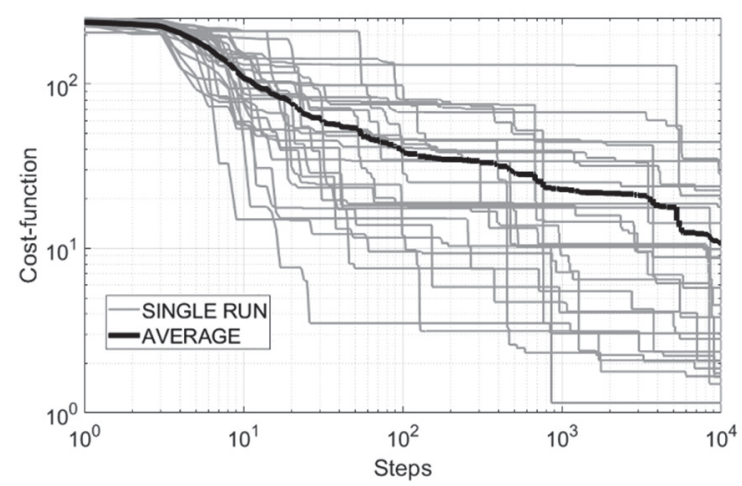

Fig. 5. Cumulative minimum of single run and the arithmetic mean (average) of all cumulative minima.

The average value of the cost-function for the best-found solution is 109.04 after 10 steps, 38.89 after 100 steps, 22.77 after 1000 steps and 10.81 after 10000 steps. The presented results confirm that the algorithm converges fast, the optimization is carried out using the same parameter values, with just two slight modifications. Namely, mutation probability is not constant and termination condition refers to the number of steps with no improvement of the solution, as it is described in the following section.

\section{MicrowaVe FiLter OptimizATION}

It takes about one second to calculate the optimization function 5000 times (the number of calculations per 25 algorithm steps for $\lambda=100$ ) on the desktop computer (Intel Core i5-6500 CPU@3.20 GHz machine with 16 GB of RAM). Note that the implemented code uses only one thread therefore its efficiency can be further improved by implementing a parallelized version. On the used computer, the complete search of the optimization space would require more than $10^{5}$ years.

The starting value of the parameter $p$ is set to $p=0.1$, while the values of the parameters $c$ and $\lambda$ are constant: $c=0.5$ and $\lambda=100$. The optimization is terminated after 
50000 successive steps if the solution is not improved. If there is no improvement in the population for 10000 steps, mutation probability is increased to 0.35 in order to explore the larger neighborhood of the parent solution in the optimization space. In the case of a better offspring being generated, this parameter $p$ is reset to $p=0.1$. The average run time of the optimization is around an hour.

We run the algorithm 20 times with random starting points. Therefore, the complete optimization procedure takes about one day for the presented results. The result of each run of the described algorithm is one of numerous suboptimal solutions in the design space.

In Fig. 6 the modulus of reflection coefficient $\left(s_{11}\right)$ is shown and in Fig. 7 the modulus of transmission coefficient $\left(s_{21}\right)$ is shown. The values of the filter components that correspond to each of 20 solutions are listed in Table 1 (values of inductors) and Table 2 (values of capacitors). One of the criteria for the optimal choice of the final filter design may be the number of different components used in the design and their prices. For example, in the design 7 all 12 components are different. On the other hand, only 9 different components are used in the solution 1.

\begin{tabular}{r|r|r|r|r|r|r}
\multicolumn{6}{c}{ TABLE 1: INDUCTANCES OF THE OPTIMIZED FILTERS } \\
No. & $L_{1}$ & \multicolumn{1}{c}{$L_{2}$} & \multicolumn{1}{c}{$L_{3}$} & \multicolumn{1}{c}{$L_{4}$} & \multicolumn{1}{c}{$L_{5}$} & $L_{6}$ \\
& {$[\mathrm{nH}]$} & {$[\mathrm{nH}]$} & {$[\mathrm{nH}]$} & {$[\mathrm{nH}]$} & {$[\mathrm{nH}]$} & {$[\mathrm{nH}]$} \\
\hline $\mathbf{1}$ & 10.0 & 1.5 & 10.0 & 5.1 & 8.2 & 2.2 \\
\hline $\mathbf{2}$ & 22.0 & 1.2 & 1.5 & 8.2 & 1.5 & 1.5 \\
\hline $\mathbf{3}$ & 22.0 & 1.5 & 22.0 & 47.0 & 30.0 & 1.2 \\
\hline $\mathbf{4}$ & 15.0 & 1.5 & 27.0 & 100.0 & 1.5 & 1.5 \\
\hline $\mathbf{5}$ & 5.1 & 5.1 & 5.8 & 2.2 & 5.8 & 1.2 \\
\hline $\mathbf{6}$ & 10.0 & 2.2 & 5.8 & 1.2 & 10.0 & 2.2 \\
\hline $\mathbf{7}$ & 10.0 & 1.5 & 22.0 & 47.0 & 15.0 & 1.2 \\
\hline $\mathbf{8}$ & 22.0 & 1.2 & 1.5 & 30.0 & 5.1 & 5.8 \\
\hline $\mathbf{9}$ & 8.2 & 1.5 & 47.0 & 150.0 & 10.0 & 5.1 \\
\hline $\mathbf{1 0}$ & 22.0 & 2.2 & 5.8 & 22.0 & 22.0 & 1.2 \\
\hline $\mathbf{1 1}$ & 10.0 & 2.2 & 2.2 & 150.0 & 1.5 & 1.5 \\
\hline $\mathbf{1 2}$ & 10.0 & 10.0 & 5.8 & 1.2 & 27.0 & 2.2 \\
\hline $\mathbf{1 3}$ & 1.2 & 5.1 & 39.0 & 15.0 & 22.0 & 8.2 \\
\hline $\mathbf{1 4}$ & 15.0 & 2.2 & 10.0 & 8.2 & 10.0 & 30.0 \\
\hline $\mathbf{1 5}$ & 15.0 & 1.2 & 1.2 & 5.1 & 22.0 & 27.0 \\
\hline $\mathbf{1 6}$ & 15.0 & 1.2 & 22.0 & 47.0 & 15.0 & 1.5 \\
\hline $\mathbf{1 7}$ & 1.2 & 5.8 & 10.0 & 1.2 & 27.0 & 1.5 \\
\hline $\mathbf{1 8}$ & 15.0 & 100.0 & 10.0 & 2.2 & 15.0 & 1.2 \\
\hline $\mathbf{1 9}$ & 5.0 & 27.0 & 47.0 & 1.2 & 5.1 & 10.0 \\
\hline $\mathbf{2 0}$ & 10.0 & 1.2 & 22.0 & 22.0 & 10.0 & 1.5 \\
\hline & & & & & & \\
\hline
\end{tabular}

The presence of the parasitic resonances near the targeted passband can be noticed in the characteristics of designs 3, $6,10,16,17$, and 18 . It can be observed that passband of some filters $(2,4,8,12$ and 20) is wider than intended, while for some of the designs (9,13 and 19) it is too narrow. Remaining six solutions (1, 5, 7, 11, 14 and 15) meet the criteria of the optimization as well. However, in the general case the total number of satisfactory solutions obtained with repeated optimizations is problem specific. We chose the solution 1 for the final design of the band-pass filter based on the characteristics in Figs. 5 and 6, as well as on the fact that the design 1 requires the minimum number of different SMD components. This example shows that insight into suboptimal solutions might provide the information about other possible solutions that can have different (secondary) qualities, other that the minimal cost function.

\begin{tabular}{|c|c|c|c|c|c|c|}
\hline No. & $\begin{array}{c}C_{1} \\
{[\mathrm{pF}]}\end{array}$ & $\begin{array}{c}C_{2} \\
{[\mathrm{pF}]}\end{array}$ & $\begin{array}{c}C_{3} \\
{[\mathrm{pF}]}\end{array}$ & $\begin{array}{c}C_{4} \\
{[\mathrm{pF}]}\end{array}$ & $\begin{array}{c}C_{5} \\
{[\mathrm{pF}]}\end{array}$ & $\begin{array}{c}C_{6} \\
{[\mathrm{pF}]}\end{array}$ \\
\hline 1 & 2.7 & 7.0 & 30.0 & 5.6 & 30.0 & 5.6 \\
\hline 2 & 1.0 & 8.0 & 47.0 & 1.2 & 47.0 & 7.0 \\
\hline 3 & 1.0 & 5.6 & 2.0 & 2.7 & 1.0 & 7.0 \\
\hline 4 & 1.8 & 7.0 & 1.0 & 1.8 & 8.2 & 5.6 \\
\hline 5 & 22.0 & 2.7 & 1.8 & 6.8 & 47.0 & 8.2 \\
\hline 6 & 2.0 & 7.0 & 20.0 & 8.2 & 2.2 & 4.7 \\
\hline 7 & 2.2 & 6.0 & 15.0 & 2.0 & 36.0 & 7.0 \\
\hline 8 & 1.0 & 2.2 & 6.0 & 6.8 & 36.0 & 5.1 \\
\hline 9 & 1.8 & 5.1 & 0.5 & 3.6 & 12.0 & 5.1 \\
\hline 10 & 1.2 & 6.8 & 27.0 & 0.5 & 1.0 & 6.8 \\
\hline 11 & 3.6 & 1.5 & 3.9 & 10.0 & 6.0 & 1.2 \\
\hline 12 & 2.2 & 2.0 & 5.1 & 7.0 & 1.0 & 6.0 \\
\hline 13 & 1.8 & 2.0 & 33.0 & 2.2 & 10.0 & 2.7 \\
\hline 14 & 1.5 & 1.0 & 2.0 & 8.0 & 4.7 & 4.7 \\
\hline 15 & 1.8 & 8.0 & 20.0 & 0.5 & 1.2 & 2.7 \\
\hline 16 & 1.8 & 8.0 & 1.8 & 3.9 & 1.8 & 5.1 \\
\hline 17 & 5.1 & 3.9 & 7.0 & 8.2 & 1.0 & 6.8 \\
\hline 18 & 2.2 & 3.6 & 2.7 & 2.2 & 1.5 & 7.0 \\
\hline 19 & 6.0 & 2.2 & 0.5 & 6.0 & 2.2 & 1.5 \\
\hline 20 & 2.7 & 7.0 & 1.0 & 0.5 & 3.6 & 7.0 \\
\hline
\end{tabular}

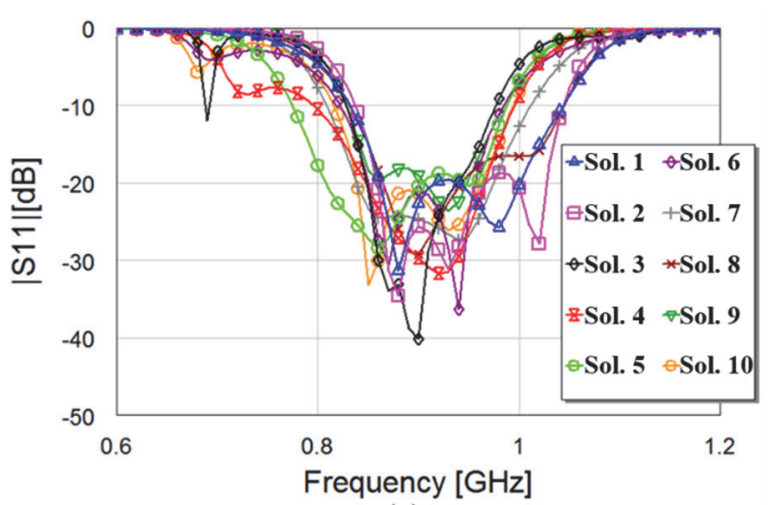

(a)

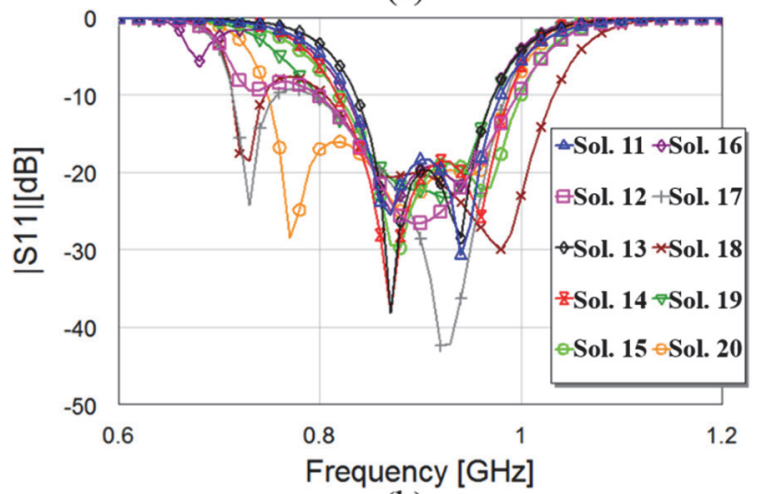

(b)

Fig. 6. The parameter $s_{11}$ of the the optimized band-pass filters: (a) solutions 1-10 (b) solutions 11-20. 


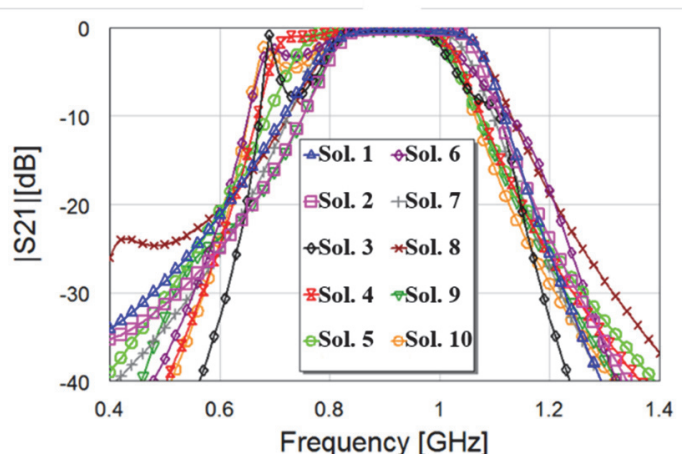

(a)

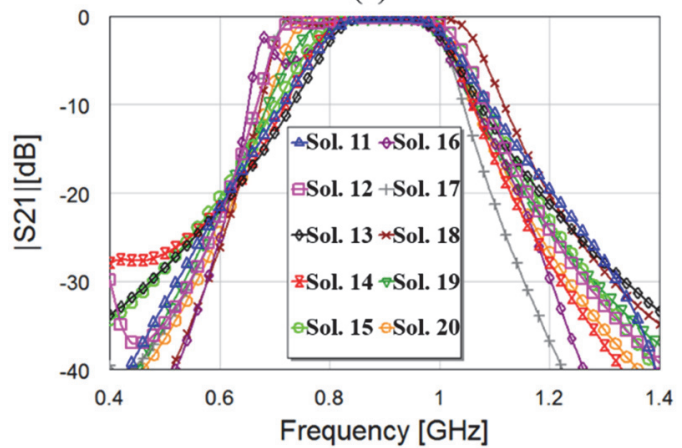

(b)

Fig. 7. The parameter $s_{21}$ of the optimized band-pass filters: (a) solutions 1-10 (b) solutions 11-20.

\section{Circuit-Solver Simulation}

In order to confirm the results of the optimization we first perform circuit-solver (2D) simulation in Microwave Office [13] of the designed filter. The model used for this simulation, shown in Fig. 8, is more detailed than the one used for the optimization.

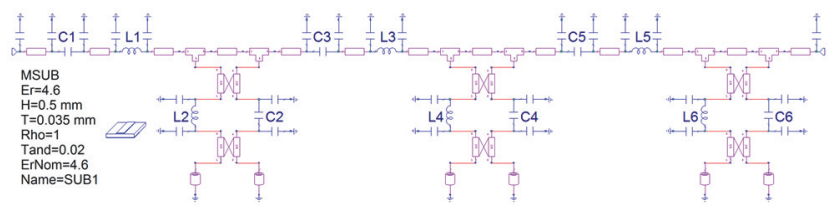

Fig. 8. Model of band-pass filter in Microwave Office.

This model includes vias, coupling between microstrip lines, T-junctions, inductors and capacitors with frequency-dependent $\mathrm{Q}$ factors of SMD components (taken from manufacturer data sheet) and additional (parasitic) capacitances due to tin droplets. The substrate loss tangent is set to 0.02 since FR-4 is used for the prototype. Results for circuit simulation of model presented in Fig. 8, overlaid with the optimized results and with Microwave Office simulation results of the starting model (based on the schematic shown in Fig. 1), are shown in Fig. 9.

Results presented in Fig. 9 are in a good agreement (the losses in the passband are increased for about $2.5 \mathrm{~dB}$ and the resonances of the filter are shifted for $2 \%$ ). The difference in $s_{21}$ in the pass-band is due to the fact that the starting model assumes the ideal (lossless) components. Although the starting model is slightly less accurate, we have used it for the optimization due to the fact that it provides faster calculations and can be coupled with used GA.

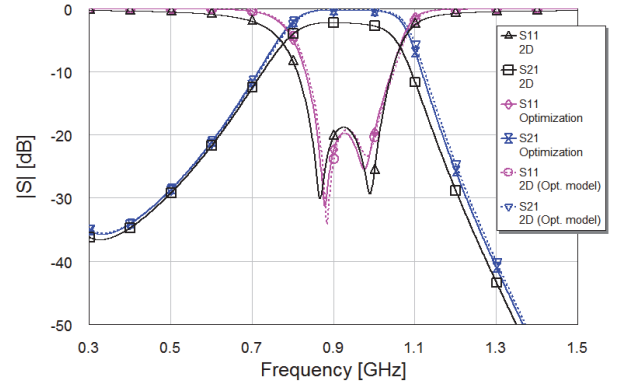

Fig. 9. The $s$-parameters of band-pass filter, 2D simulation of the detailed model, optimization results and 2D simulation of the starting model.

\section{3D SIMULATION}

With the aim of verifying the presented results further, a $3 \mathrm{D}$ model of the filter is created. The 3D model of the filter is shown in Fig. 10. A full-wave electromagnetic simulation is performed using WIPL-D Pro software [14]. The SMD components are represented as lumped loadings and the dielectric has finite dimensions. The $s$-parameters of the model simulated in WIPL-D 3D electromagnetic solver are compared to the results of the ideal model (used for the optimization) in Fig. 11.

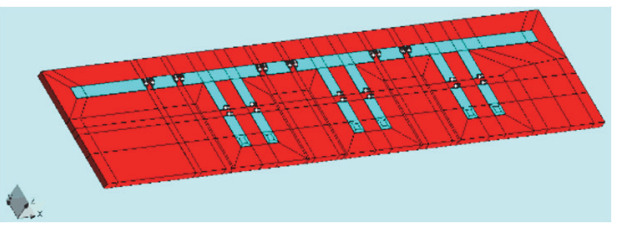

Fig. 10. 3D electromagnetic model of band-pass filter.

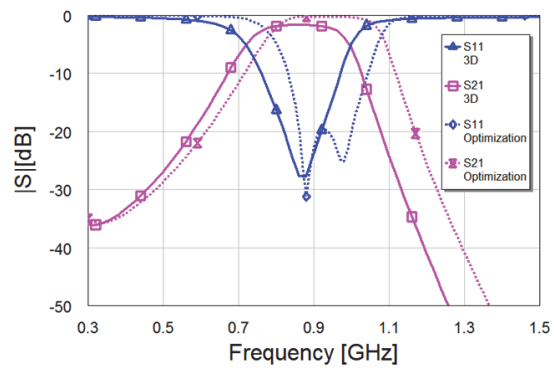

Fig. 11. The $s$-parameters of band-pass filter, 3D simulation and optimization results.

The results of a full-wave electromagnetic simulation suggest a shift in the frequency response for about $100 \mathrm{MHz}$. The 3D WIPL-D model and the 2D Microwave Office model have the same values of all parameters and the same dimensions. However, the 3D simulation takes into account the complete electromagnetic coupling, as well as the finite substrate dimension, and therefore some differences between the results in Fig. 9 and Fig. 11 are expected.

\section{Measurements of The Prototype}

The manufactured prototype is shown in Fig. 12. The measurements of the $s$-parameters of the fabricated filter were carried out using Agilent E5062A network analyzer. Obtained results, overlaid with the results of the simulations and the results of yield analysis performed in Microwave Office, are presented in Fig. 13 and Fig. 14. Yield analysis 
takes into account the tolerances of the used SMD capacitors and inductors.

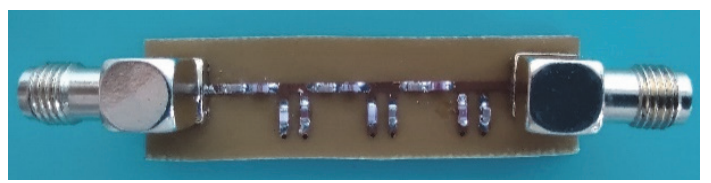

Fig. 12. Fabricated prototype of band-pass filter.

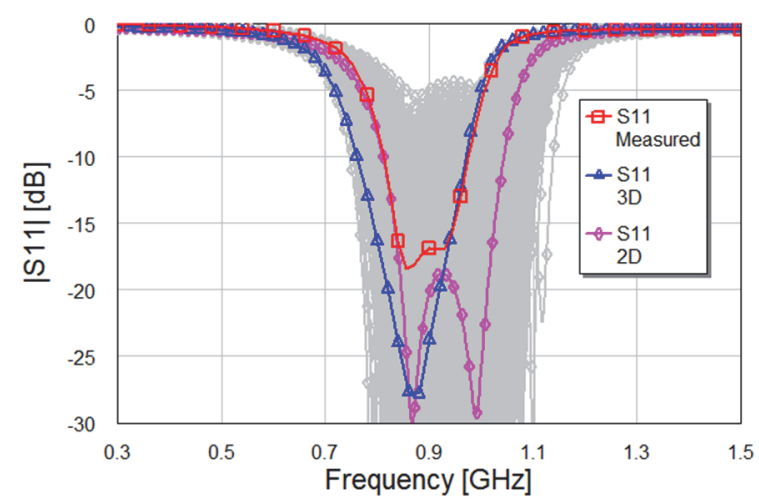

Fig. 13. The parameter $s_{11}$ of band-pass filter, measured, simulated (2D and $3 \mathrm{D})$ results and the results of yield analysis (gray).

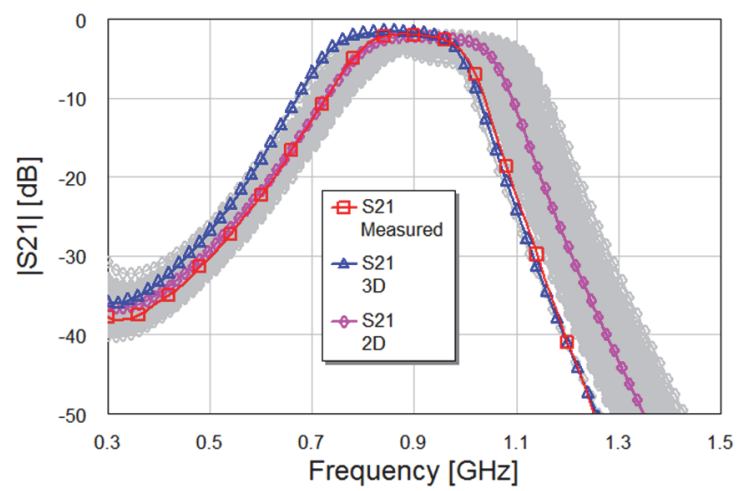

Fig. 14. The parameter $s_{21}$ of band-pass filter, measured, simulated (2D and 3D) results and the results of yield analysis (gray).

The magnitude of $s_{21}$ in the targeted passband from $850 \mathrm{MHz}$ to $950 \mathrm{MHz}$ is between $-1.9 \mathrm{~dB}$ and $-2.4 \mathrm{~dB}$, while the maximum of the magnitude of the parameter $s_{11}$ in the same band is $-14.7 \mathrm{~dB}$. We observe that both the circuit solver and the $3 \mathrm{D}$ electromagnetic simulations yield results that are up to $75 \mathrm{MHz}$ away from the measured ones. The results obtained by simulations in Microwave Office are in good agreement with the measured results along the first transition band. The results of the $3 \mathrm{D}$ simulations are in line with the measured parameters along the second transition band. Measured and simulated results differ due to parasitic effects, tolerances for components and also due to different modeling approaches. While further improvements of the models are possible, the results clearly demonstrate that the design of a filter with discrete-valued optimization is a viable design approach.

\section{CONCLUSION}

We presented a design approach of the lumped-element microwave band-pass filter based on optimization using only discrete component values. Considering that the simulations of $2 \mathrm{D}$ and $3 \mathrm{D}$ models of the filter provide good agreement with the results of the initial optimization using GA, the prototype is fabricated without additional tuning and further adjustments of component values. Simulated results predict a filter bandwidth that is about $75 \mathrm{MHz}$ wider than the measured ones, which is expected due to tolerances of the used components.

\section{REFERENCES}

[1] V. Petrović, D. Tošić, A. Djordjević, Microwave passive circuits Univ. of Belgrade, Serbia: School of Electrical Engineering, 2010.

[2] A. Djordjević, D. Tošić, Microwave technique. University of Belgrade, Serbia: School of Electrical Engineering, 2010.

[3] M. Ilić, S. Savić, Microwave electronics. University of Belgrade, Serbia: School of Electrical Engineering, Academic mind, 2016.

[4] D. Pozar, Microwave Engineering, Wiley, New Jersey, 2011.

[5] R. J. Cameron, C. M. Kudsia, R. R. Mansour, Microwave Filters for Communication Systems: Fundamentals, Design, and Applications. New Jersey: John Wiley \& Sons, 2018.

[6] J.-S. Hong, Microstrip filters for RF/microwave applications. New Jersey: John Wiley \& Sons, 2011.

[7] D. Ninković and D. Olćan, "Genetic Algorithm Applied to Discrete Optimization of Band-Pass Microwave Filter," 2020 28th Telecommunications Forum (TELFOR), Belgrade, Serbia, 2020, pp. 1-4, doi: 10.1109/TELFOR51502.2020.9306577.

[8] M. Ferber et al., "Discrete optimization of EMI filter using a genetic algorithm," 2014 International Symposium on Electromagnetic Compatibility, Tokyo, Tokyo, 2014, pp. 29-32.

[9] J. Lowe, L. Levesque, E. O'Dell and L. Dunleavy, "Enabling Discrete Optimization of Surface Mount Component Values in Microwave Circuit Design," 2019 IEEE 20th Wireless and Microwave Technology Conference (WAMICON), Cocoa Beach, FL, USA, 2019, pp. 1-5.

[10] J. Kahler, "Complete Filter Design with Discrete Elements Made Easy", Microwaves and RF, February 2019.

[11] D. Olćan, Lecture Notes, Optimization algorithms in engineering, University of Belgrade, Serbia: School of Electrical Engineering, 2019.

[12] B. Doerr, C. Doerr, F. Ebel, "From black-box complexity to designing new genetic algorithms," Theoretical Computer Science, vol. 567, pp. 87-104, Elsevier, 2015.

[13] Cadence AWR Microwave Office, https://www.awr.com/awrsoftware/products/microwave-office.

[14] WIPL-D Pro, https://wipl-d.com/products/wipl-d-pro

[15] K. F. Man, K. S. Tang and S. Kwong, "Genetic algorithms: concepts and applications", IEEE Trans. Industrial Electron., vol. 43, pp. 519 534, Oct. 1996.

[16] D.E. Goldberg, Genetic Algorithms in Search, Optimization, and Machine Learning, Addison-Wesley Professional, 1989. 